\title{
Revisiting the role of magnetic resonance imaging in the characterization of focal hepatic lesions
}

\author{
Revisitando o papel da ressonância magnética na caracterização das lesões hepáticas focais
}

Daniel Nóbrega da Costa*

In the original article "Differentiation between simple cyst and hepatic hemangioma utilizing T2weighted magnetic resonance imaging with gradientecho (b-FFE) technique", included in the present issue of Radiologia Brasileira, the authors open the discussion of an important subject.

Since the 1980's, with the widened utilization of magnetic resonance imaging (MRI) in the medical practice, this new imaging modality brought a series of inherent peculiarities, assuring its place in the radiological world. Almost always compared with computed tomography - whose major qualities are the efficiency (fast imaging and with plenty useful information) and consistency (the method can be utilized in non-collaborative patients and with a system that is operationally less complex, thus resulting in images less vulnerable to technical difficulties), characteristics that place this method in the top position as the major MRI competitor among the sectional imaging methods -, MRI stands out by its capacity of differentiating tissues like any other modality.

In this context, the investigation of focal hepatic lesions deserves special attention. Both in cases of asymptomatic individuals, as an incidental finding, and in patients with a relevant previous clinical history (for example, a known malignant neoplasm, chronic hepatopathy, etc.), the characterization of focal hepatic lesions is as much essential as their detection, considering that the nature of the lesion will determine the clinical conduct to be adopted.

\footnotetext{
* MD, Physician Assistant, Department of Imaging Diagnosis at Instituto do Câncer do Estado de São Paulo (ICESP) and Instituto de Radiologia do Hospital das Clínicas da Faculdade de Medicina da Universidade de São Paulo (InRad/HC-FMUSP), São Paulo, SP, Brazil. E-mail: dnobrega@gmail.com
}

Amongst the wide spectrum of entities included in the differential diagnosis of focal hepatic lesions, cysts and hemangiomas account for a significant number of cases. Although the practical utility of differentiating between two benign entities may be questioned, particularly in cases of small lesions with no potential for complications, any study validating the capacity of a method to offer information on the nature of the tissues should be positively considered. This fact gains particular importance in a scenario where the safety in the utilization of paramagnetic contrast agents is under debate, demanding efforts and attention from the radiological community in order to optimize and extract the maximum information possible from non-contrastenhanced studies.

While some groups are involved in the research of new approaches such as the utilization of new contrast agents (superparamagnetic iron oxide) or alternative pulse sequences (such as diffusion sequences and spectroscopy), other groups are re-evaluating the diagnostic accuracy of the method and trying to optimize the utilization of the already widely known sequences. This is the case of the original article included in the present issue of $\mathbf{R B}$, where the authors have designed a prospective, observational investigation demonstrating the utility of the b-FFE sequence in the routine evaluation of the liver by MRI, specifically as regards the differentiation between cysts and hemangiomas. According to the authors, the advantages of this pulse sequence include reduced acquisition time and immunity to motion artifacts. Additionally the study demonstrated the simplicity of the method corroborating the short learning curve for interpretation of these findings, with an optimum intra- and interobserver agreement, a quite positive result in any study. On the other hand, it is 
important to note that patients with a previous history of malignant neoplasm - a subgroup that should expectedly benefit from the characterization of focal hepatic lesions - were excluded from the sample. Another topic to be considered is the subjective character prevalent in the evaluation of these lesions. This approach is frequently adopted in the clinical practice, but may raise criticism in the scientific environment although partially softened by the already mentioned high intra- and interobserver agreement. While several strategies are aimed at a higher efficiency in the radiologists' work flow, a topic deserving attention is the fact that the majority of studies in the literature suggest the addition of pulse sequences in the protocols utilized, instead of replacing the sequences currently utilized by other more efficient sequences, thus requiring longer images acquisition time (higher number of sequences) and longer time for images interpretation (higher number of images).

Based on these points, it can be conclude that further studies will be useful for validating the significant findings described in the mentioned original article, and possibly progressing in the differential diagnosis with a higher clinical impact: the evaluation of a particular lesion aggressiveness (going beyond the traditional "benign versus malignant", by speculating about the malignancy degree of this lesion), and the identification of radiological signs which allow the indication of the more effective therapeutic strategy for each situation (indicating to oncologists with patients presenting hepatic metastasis, for example, the prospective response of a specific lesion to drugs of a determined pharmacological group). 